\title{
Abstraction in Probabilistic Process Algebra
}

\author{
S. Andova and J.C.M. Baeten \\ Department of Computing Science \\ Eindhoven University of Technology, The Netherlands \\ e-mail: \{suzana, josb\}@win.tue.nl, fax: +31 (0)402475361
}

\begin{abstract}
Process algebras with abstraction have been widely used for the specification and verification of non-probabilistic concurrent systems. The main strategy in these algebras is introducing a constant, denoting an internal action, and a set of fairness rules. Following the same approach, in this paper we propose a fully probabilistic process algebra with abstraction which contains a set of verification rules as counterparts of the fairness rules in standard $A C P$-like process algebras with abstraction. Having probabilities present and employing the results from Markov chain analysis, these rules are expressible in a very intuitive way. In addition to this algebraic approach, we introduce a new version of probabilistic branching bisimulation for the alternating model of probabilistic systems. Different from other approaches, this bisimulation relation requires the same probability measure only for specific related processes called entries. We claim this definition corresponds better with intuition. Moreover, the fairness rules are sound in the model based on this bisimulation. Finally, we present an algorithm to decide our branching bisimulation with a polynomial-time complexity in the number of the states of the probabilistic graph.
\end{abstract}

\section{Introduction and Motivation}

In this work we treat the problem of abstraction from internal actions in fully probabilistic process algebra and its model based on branching bisimulation. One of the motives to introduce probabilities in formal methods is that they can be used to model fairness. Since the idea of fairness rules ( 5 ) together with abstraction (introduced by the abstraction operator $\tau_{I}$ and a constant $\tau$ denoting an internal action) is central to the verification techniques in process algebra we introduce verification rules in fully probabilistic process algebra that arise rather in a natural way from the ones defined in standard process algebra. These rules express the idea that due to a non-zero probability for a system to execute an external action, abstraction from internal steps will yield the external step(s) with probability 1 after finitely many repetitions. For example, if one process can execute external action $a$ with probability $\pi$, external action $b$ with probability $\rho$ and with probability $1-\pi-\rho$ after executing an internal action it behaves the same as initially, then it is clear that the probability to perform the internal step infinitely many times is equal to 0 , or in other words, the probability to perform either $a$ or $b$ eventually is 1 . Next, the question arises: "With what probability

T. Margaria and W. Yi (Eds.): TACAS 2001, LNCS 2031, pp. 204-214 2001

(C) Springer-Verlag Berlin Heidelberg 2001 
$a$ (resp. $b$ ) occurs?". 9 gives the answer to this question as: the probability of $a$ is $\pi /(\pi+\rho)$ and the probability of $b$ is $\rho /(\pi+\rho)$. This corresponds to the absorption probabilities for the Markov chain given in Figure 11. In our theory the notion that relates these two processes can be easily expressed with the following verification rule: if $X=a 女_{\pi} b \nvdash_{\rho} i \cdot X$ (where $i$ is an internal action), then $\tau \cdot \tau_{\{i\}}(X)=\tau \cdot\left(a \uplus_{\pi /(\pi+\rho)} b\right)$. (For more details of the semantics see 2 and 7.) A reader familiar with process algebra can easily see the resemblance with the $K F A R_{1}^{b}$ rule ( 7 ) with non-deterministic choice replaced by probabilistic choice.

a)

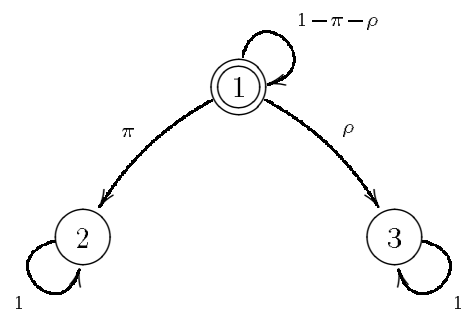

b)

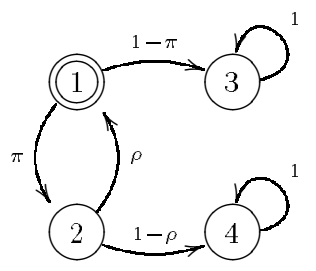

Fig. 1. Absorbing Markov chains.

Proceeding with similar reasoning for the more complex rule $K F A R_{2}^{b}$ we achieve a situation in which the definition of weak (branching) bisimulation proposed in 9 cannot abstract away the internal cycle. But working with recursive equations in our process algebra we can introduce a counterpart of this rule in the probabilistic setting in the following way:

$$
\begin{gathered}
X_{1}=i \cdot X_{2} \uplus_{\pi} Y_{1} \\
X_{2}=i \cdot X_{1} \uplus_{p} Y_{2}, I=\{i\} \\
\hline \tau \cdot \tau_{I}\left(X_{1}\right)=\tau \cdot\left(\tau_{I}\left(Y_{1}\right) \uplus_{\alpha} \tau_{I}\left(Y_{2}\right)\right)
\end{gathered}
$$

where $X_{1}$ is the root variable and $\alpha=(1-\pi) /(1-\pi \rho)$. The transition systems (as defined in 9) for these processes are given in Figure 2 (for the sake of simplicity the initial internal steps are not shown) and the corresponding Markov chain of the first process is shown in Figure 11 . The values of probabilities $\alpha$ and $\beta=1-\alpha=\pi(1-\rho) /(1-\pi \rho)$ are obtained as the absorption probabilities when $X_{1}$ is the root variable, that is, 1 is "the initial state of the system" (in

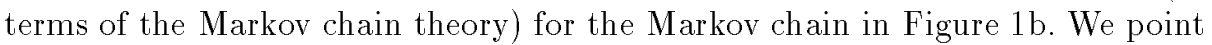
out that the absorption probabilities for this system differ for various initial distributions.

Further on, we define a probabilistic branching bisimulation relation on the set of fully probabilistic graphs that abstracts away internal actions in wider variety of cases than the definition in 9 . It will turn out that the set of graphs modulo this relation gives rise to a model of our process algebra. Two nodes are considered bisimilar if they have the same branching structure and if taken as roots they have the same probability measures. This means that if the system is in either of these states then the probabilities to execute a visible action and also the probabilities to enter with an internal step into a different equivalence 
a)

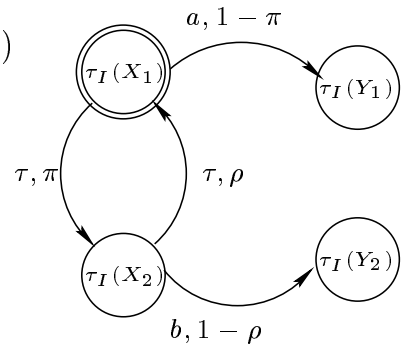

b)

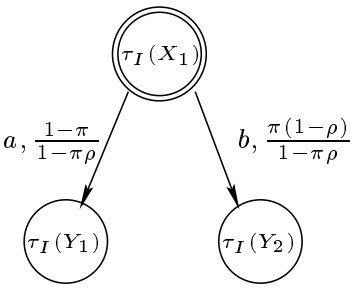

Fig. 2. Related fully probabilistic processes.

class are the same. In this definition the notion of an entry plays a central role (a notion to be formally defined in Definition 10 on page 2 2 . Starting from the roots of the graphs we build a set of entries for which the probability measure is checked in the next stage. Informally, we can say that a node not found as an entry is just involved in (is just a part of) an internal path (or a cycle) that starts in an entry with the same branching structure as the non-entry node; the node is not involved in any other path. Also, after execution of a visible action the system never goes to the state interpreted by this non-entry node. For example, in the graph in Figure $21, \tau_{I}\left(X_{2}\right)$ is a non-entry node (no path from $\tau_{I}\left(Y_{1}\right)$ or $\tau_{I}\left(Y_{2}\right)$ goes back to $\left.\tau_{I}\left(X_{2}\right)\right)$.

Moreover, in the paper we give an algorithm which decides the probabilistic branching bisimulation in polynomial time in the number of states of the probabilistic graph.

Because parallel composition based on interleaving (see e.g. 2.3 ) includes non-determinism, this algebra and semantics cannot deal with such parallel composition. However, since we define the branching bisimulation on the alternating model, and we also consider the non-deterministic (or action) nodes in our definition of bisimulation, we expect that the extension with non-deterministic choice can be achieved on the basis of the results presented here. This will enable the extension with interleaving parallel composition.

Motivating example. In order to depict the idea of our approach we give the following motivating example. An experimenter has two coins $A$ and $B$. $A$ is a fair coin with the probability distribution $\{1 / 2$ head, $1 / 2$ tail $\}$, and $B$ is biased with distribution $\{1 / 3$ head, $2 / 3$ tail $\}$. First he throws coin $A$. If head turns up the throwing is over and he announces "head". If tail shows up then he throws coin $B$. If tail turns up then the throwing is over and he announces "tail", but if head turns up then he takes coin $A$ and performs the experiment again. The process can be specified by the following recursive specification:

$A=$ tail $_{A} \cdot B \uplus_{1 / 2}$ head $_{A} \cdot$ sayhead

$B=$ head $_{B} \cdot A \bigsqcup_{1 / 3}$ tail $_{B} \cdot$ saytail

where sayhead and saytail are atomic actions expressing the observable events of announcing "head" and "tail", respectively. Abstracting from head ${ }_{A}$, tail $_{A}$, head $_{B}$ and $\operatorname{tail}_{B}$ and applying the rule PVR2 we obtain that the probability to end the 
experiment by saying "head" or "tail", is $3 / 5$ and $2 / 5$, respectively. We point out that coin $A$ was chosen as the initial coin. We can imagine that the throwing is performed in an isolated room and an observer can hear only the final outcome, but for her it is not clear what kind of experiment is performed in the room.

The observer will make the same observation if the experimenter performs some other experiment. Namely, instead of two coins he has only one fair die. He rolls it and if the outcome is 1 he rolls it again. If the outcome is an even number he announces "head" and if the outcome is 3 or 5 he announces "tail".

Related Work We have already mentioned the relation of this paper with 9. Namely, the branching (weak) bisimulation for fully probabilistic systems presented in 9 is finer than ours. Reasoning in the process algebra which we present here, with the rules $P V R 1, P R V 2, \ldots$ and speaking informally, their bisimulation defines just a model of $P V R 1$, not of $P V R 2$ and more complex rules (see further on).

Similarities of the bisimulation defined here can be found with the branching bisimulation of Jou in 11 . The proposed probabilistic branching bisimulation in the latter work is defined on the set of finite trees. And the author's attention is more focused on the axiom: $a\left(\tau\left(x \uplus_{\pi} y\right) \uplus_{\rho} y\right)=a\left(x \uplus_{\pi \rho} y\right)$ and not on any rules that treat internal cycles. Our definition of bisimulation coincides with his one on the set of finite trees (in terms of process algebra, the set of closed terms). And the branching bisimulation presented in this paper is an extension of his branching bisimulation over infinite processes.

\section{Definitions and Results}

In 2 a probabilistic process algebra containing both probabilistic choice and non-deterministic choice is introduced. Our current work is based on a subalgebra of that one for which non-deterministic choice has been excluded. Having both choices and abstraction at the same time leads to a more complex axiomatization and this extension, we think, can be achieved on the basis of the definitions we give here. Due to the absence of non-determinism the interleaving parallel composition as treated in 2 cannot be incorporated in this fully probabilistic process algebra. On the other hand, some version of synchronous parallel composition may be considered in such a process algebra (also see 6 ).

In addition to the set of atomic actions $A$ and the constant $\tau$ the fully probabilistic process algebra presented here has three operators: the probabilistic choice operator $\uplus_{\pi}, \pi \in\langle 0,1\rangle$, the sequential composition - and the abstraction operator $\tau_{I}$ for $I \subseteq A$. The axiom system is given in Table $\mathbf{\|}$ and $\boldsymbol{\nabla}$ Informally, process $x \forall_{\pi} y$ behaves as $x$ with probability $\pi$ and as $y$ with probability $1-\pi$. Also, process $x \square_{\pi} y \uplus_{\rho} z$ behaves as $x$ with probability $\pi$, as $y$ with probability $\rho$ and as $z$ with probability $1-\pi-\rho$. This algebra will be denoted by $\operatorname{pr} B P A_{\tau}$. We also add to the algebra a set of verification rules PVR1 and PVRn for $n \geq 2$ : $X_{1}=i \cdot X_{1} \uplus_{\pi_{1}} Y_{1}, \tau \neq i \in I$

$\tau \cdot \tau_{I}\left(X_{1}\right)=\tau \cdot \tau_{I}\left(Y_{1}\right)$ 


$$
\begin{aligned}
& X_{1}=i_{1} \cdot X_{2} \uplus_{\pi_{1}} Y_{1} \\
& X_{2}=i_{2} \cdot X_{3} \uplus_{\pi_{2}} Y_{2} \\
& \cdot \\
& X_{n-1}=i_{n-1} \cdot X_{n} \uplus_{\pi_{n-1}} Y_{n-1} \\
& X_{n}=i_{n} \cdot X_{1} \uplus_{\pi_{n}} Y_{n}, I \cup\{\tau\} \supseteq\left\{i_{1}, i_{2}, \ldots, i_{n}\right\} \neq\{\tau\} \\
& \tau \cdot \tau_{I}\left(X_{1}\right)=\tau \cdot\left(\tau_{I}\left(Y_{1}\right) \uplus_{\alpha_{1}} \tau_{I}\left(Y_{2}\right) \uplus_{\alpha_{2}} \ldots \uplus_{\alpha_{n-2}} \tau_{I}\left(Y_{n-1}\right) \uplus_{\alpha_{n-1}} \tau_{I}\left(Y_{n}\right)\right)
\end{aligned}
$$

\begin{tabular}{|c|c|c|}
\hline$(x \cdot y) \cdot z$ & $=x \cdot(y \cdot z)$ & $A 5$ \\
\hline$\square_{\pi} y$ & $=y \varpi_{1-\pi} x$ & 1 \\
\hline$x \square_{\pi}\left(y \square_{\rho} z\right.$ & $=\left(x \square_{\frac{\pi}{\pi+\rho-\pi \rho}} y\right.$ & $\operatorname{Pr} A C 2$ \\
\hline $\begin{array}{l}\square_{\pi} x \\
\left.x \square_{\pi} y\right) \cdot z\end{array}$ & $\begin{array}{l}=x \\
=x \cdot z \square_{\pi} y \cdot z\end{array}$ & \\
\hline
\end{tabular}

where $\alpha_{1}=\frac{1-\pi_{1}}{1-\pi_{1} \cdot \pi_{2} \cdots \cdot \pi_{n}}, \alpha_{j}=\frac{\pi_{1} \cdots \pi_{j-1} \cdot\left(1-\pi_{j}\right)}{1-\pi_{1} \cdots \cdot \pi_{n}}$ for $j: 1<j \leq n$ and $\pi_{k} \in\langle 0,1\rangle$ for $k: 1<k<n$. If we refer to the algebra extended with these rules we write $p r B P A_{\tau}+P \bar{V} R 1+P V R 2+\ldots$

Table 1. Axioms for probabilistic choice and sequential composition.

\begin{tabular}{llll}
\hline$x \cdot \tau$ & $=x$ & & $T 1$ \\
& & & \\
$\tau_{I}(\tau)$ & $=\tau$ & & \\
$\tau_{I}(a)$ & $=a$ & if $a \notin I$ & $T I 1$ \\
$\tau_{I}(a)$ & $=\tau$ & if $a \in I$ & $T I 2$ \\
$\tau_{I}(x \cdot y)$ & $=\tau_{I}(x) \cdot \tau_{I}(y)$ & & $T I 4$ \\
$\tau_{I}\left(x \square_{\pi} y\right)$ & $=\tau_{I}(x) \varpi_{\pi} \tau_{I}(y)$ & & $\operatorname{Pr} T I$
\end{tabular}

Table 2. Axioms for abstraction $\left(I \subseteq A_{\tau}\right)$.

The reader has noticed that we use a set of equations of the form $X_{j}=P_{j}, j=$ $1, \ldots, n$, to specify recursive behaviour. In the above equations, $X_{1}, \ldots, X_{n}$ are pairwise distinct variables and $P_{1}, \ldots, P_{n}$ are guarded terms over the given signature (see e.g. 7 ). Every recursive specification has a root variable. In the verification rule PVRn, $X_{1}$ is the root variable of the recursive specification.

In order to construct a model of this algebra we introduce fully probabilistic graphs. Further on, we define probabilistic branching bisimulation. We work in the alternating model with two types of nodes (processes): probabilistic nodes with probabilistic outgoing transitions only, denoted by $\leadsto$, and action nodes with action transitions only, denoted by $\stackrel{a}{\rightarrow}$, for $a \in A_{\tau}$. By allowing at most one action transition to leave an action node we obtain the alternating model of fully probabilistic process algebra.

Definition 1. Let $A$ be a countable set of atomic actions. A fully probabilistic graph $g$ is a tuple $\left(S_{p} \cup S_{n} \cup\{N I L\}, \leadsto, \rightarrow, \mu\right.$, root) consisting of:

- a countable set $S_{p}$ of probabilistic states,

- a countable set $S_{n}$ of action states such that $S_{p} \cap S_{n}=\emptyset$ and NIL $\notin S_{p} \cup S_{n}$, 
- root $\in S_{p}$,

- a relation $\sim \subseteq S_{p} \times S_{n}$

- a function $\rightarrow: S_{n} \mapsto S_{p} \cup\{N I L\} \times A_{\tau}$, and

- a partial function $\mu: S_{p} \times S_{n} \mapsto\langle 0,1]$ such that $\mu(p, n)$ is defined iff $(p, n) \in \sim$ for $(p, n) \in S_{p} \times S_{n}$ and for any $p \in S_{p}, \sum_{n \in S_{n}} \mu(p, n)=1$.

We denote $S=S_{p} \cup S_{n}$. If $S$ is a finite set then we say that the probabilistic graph $g$ is finite. NIL is called the terminating state. If $N I L$ is not reachable from the root of $g$ then it can be ignored. Function $\mu$ is called the probability distribution function of $g$.

If $(p, n) \in \sim$, we write $p \leadsto n$. If $\rightarrow(n)=(p, a)$ we write $n \stackrel{a}{\rightarrow} p$. For sake of simplicity, instead of writing the value of function $\mu$ separately, if $p \leadsto n$ we write $p \stackrel{\mu(p, n)}{\sim} n$. By $\mathbf{G}$ we denote the set of all finite fully probabilistic graphs.

Note: If $\rightarrow$ is not a function from $S_{n}$ to $S_{p} \cup\{N I L\} \times A_{\tau}$, but a subset of $S_{n} \times S_{p} \cup\{N I L\} \times A_{\tau}$, we get the general class of probabilistic graphs, including non-determinism. In this case action nodes are rather called non-deterministic nodes, as we do in 23.

Definition 2. Let $g=(S \cup\{N I L\}, \sim, \rightarrow, \mu$, root $)$ be a fully probabilistic graph We say that $g$ is root acyclic if there is no node $n \in S_{n}$ and $a \in A_{\tau}$ such that $n \stackrel{a}{\rightarrow}$ root. Otherwise we say that $g$ is root cyclic.

We define the root unwinding map $\rho: \mathbf{G} \mapsto \mathbf{G}$ as follows:

- if $g$ is root acyclic, then $\rho(g)=g$;

- if $g$ is root cyclic, then $\rho(g)=\left(S \cup\{N I L\} \cup\{\right.$ newroot $\}, \sim^{\prime}, \rightarrow, \mu^{\prime}$, newroot $)$, where newroot is a new node, $\sim^{\prime}=\sim \cup\{($ newroot,$n):$ root $\leadsto n\}$ and

$$
\mu^{\prime}(p, n)= \begin{cases}\mu(p, n) & \text { if } p \in S \\ \mu(\text { root }, n) & \text { if } p=\text { newroot }\end{cases}
$$

Proposition 1. Let g be a fully probabilistic graph.

$i$. Then $\rho(g)$ is root acyclic.

ii. $g \leftrightarrow \rho(g)$, that is, $g$ and $\rho(g)$ are strongly bisimilar.

\section{Interpretation of Constants and Operators in G}

Definition 3. (Interpretation of the constants) If $a \in A_{\tau}$, its interpretation is $[a]=\left(\left\{s_{p}\right\} \cup\left\{s_{n}\right\} \cup\{N I L\},\left\{s_{p} \leadsto s_{n}\right\},\left\{s_{n} \stackrel{a}{\rightarrow} N I L\right\}, \mu\left(s_{p}, s_{n}\right)=1, s_{p}\right)$.

Definition 4. (Interpretation of the operators) Let $g$ and $h$ be graphs in $\mathbf{G}$ and $g=\left(S_{g} \cup\left\{N I L_{g}\right\}, \sim g, \rightarrow_{g}, \mu_{g}, \operatorname{root}_{g}\right)$ and $h=\left(S_{h} \cup\left\{N I L_{h}\right\}, \sim \rightarrow_{h}, \rightarrow_{h}, \mu_{h}, \operatorname{root}_{h}\right)$.

Sequential composition: $g \cdot h$ is defined as:

$$
\left(S_{g} \cup S_{h} \cup\left\{N I L_{h}\right\}, \sim g \cup \sim h, \rightarrow, \mu, \operatorname{root}_{g}\right),
$$

where: $\rightarrow=\left(\rightarrow_{g} \backslash\left\{n \stackrel{a}{\rightarrow}\right.\right.$ NIL $\left.\left.L_{g}: n \in S_{g}, a \in A c t_{\tau}\right\}\right) \cup \rightarrow_{h}$

$$
\cup\left\{n \stackrel{a}{\rightarrow} \operatorname{root}_{h}: n \in S_{g}, a \in \text { Act }_{\tau}, n \stackrel{a}{\rightarrow} N I L_{g}\right\},
$$


and $\quad \mu(p, n)= \begin{cases}\mu_{g}(p, n) & \text { if } p, n \in S_{g} \\ \mu_{h}(p, n) & \text { if } p, n \in S_{h}\end{cases}$

Probabilistic choice: $g \uplus_{\pi} h$, for $\pi \in\langle 0,1\rangle$, is defined as:

$$
(S \cup\{N I L\}, \leadsto, \rightarrow, \mu, \text { root }),
$$

where: $S=\left(S_{g} \backslash\left\{\right.\right.$ root $\left.\left._{g}\right\}\right) \cup\left(S_{h} \backslash\left\{\right.\right.$ root $\left.\left._{h}\right\}\right) \cup\{$ root $\}$, root $\notin S_{g} \cup S_{h}$,

$\leadsto=\left(\sim g \backslash\left\{\right.\right.$ root $\left.\left._{g} \leadsto n: n \in S_{g}\right\}\right) \cup\left(\sim h \backslash\left\{\right.\right.$ root $\left.\left._{h} \leadsto n: n \in S_{h}\right\}\right)$

$\cup\left\{\right.$ root $\sim n: n \in S_{g}$, root $\left._{g} \sim n\right\} \cup\left\{\right.$ root $\sim n: n \in S_{h}$, root $\left._{h} \sim n\right\}$,

$\rightarrow=\rightarrow_{g} \cup \rightarrow_{h}$ with the remark that $N I L_{g}$ and $N I L_{h}$ are identified and this node is named NIL,

and $\quad \mu(p, n)= \begin{cases}\mu_{g}(p, n) & \text { if } p, n \in S_{g} \backslash\left\{\operatorname{root}_{g}\right\} \\ \mu_{h}(p, n) & \text { if } p, n \in S_{h} \backslash\left\{\operatorname{root}_{h}\right\} \\ \pi \cdot \mu_{g}\left(\operatorname{root}_{g}, n\right) & \text { if } p=\operatorname{root} \& n \in S_{g} \& \operatorname{root}_{g} \sim n \\ (1-\pi) \cdot \mu_{h}\left(\operatorname{root}_{h}, n\right) & \text { if } p=\operatorname{root} \& n \in S_{h} \& \operatorname{root}_{h} \sim n\end{cases}$

Abstraction: $\tau_{I}(g)$ for $I \subseteq A$ is defined as:

$$
\left(\bar{S}_{g} \cup\left\{N I L_{g}\right\}, \sim g, \rightarrow, \mu_{g}, \operatorname{root}_{g}\right),
$$

where: $p \stackrel{a}{\rightarrow} n$ iff $p \stackrel{a}{\rightarrow} n$ and $a \notin I$ and

$p \stackrel{\tau}{\rightarrow} n$ iff $p \stackrel{a}{\rightarrow}_{g} n$ and $a \in I \cup\{\tau\}$.

Similarly to the non-probabilistic version of bisimulation relations including silent steps (in particular branching bisimulation) we allow here an observable action $a(a \neq \tau)$ to be simulated by a sequence of transitions such that exactly the last transition is an $a$-transition and the rest are internal transitions inside the same equivalence class. The new problem we should think about is the way we calculate the probability measure of such a sequence of transitions according to the probability distribution function $\mu$. For that reason we sketch (repeat) the standard concept used to define a probability measure (see 89 ), adapted for the alternating model of fully probabilistic systems.

Let $g=\left(S_{p} \cup S_{n} \cup\{N I L\}, \leadsto, \rightarrow, \mu\right.$, root $)$ be a finite fully probabilistic graph.

Definition 5. For $p \in S_{p}, n \in S_{n}, C \subseteq S_{p} \cup\{N I L\}$ and $a \in A_{\tau}$ we define:

- $n \stackrel{a}{\rightarrow} C$ iff $\exists q \in C: n \stackrel{a}{\rightarrow} q$;

- $\mathbf{P}(p, a, C)=\sum_{n: n \rightarrow C} \mu(p, n)$ and $\mathbf{P}(p, a, q)=\mathbf{P}(p, a,\{q\}) ;$

- An execution fragment or finite path is a nonempty finite sequence

$$
\sigma=p_{0} \leadsto n_{0} \stackrel{a_{1}}{\rightarrow} p_{1} \leadsto n_{1} \stackrel{a_{2}}{\rightarrow} p_{2} \ldots p_{k-1} \leadsto n_{k-1} \stackrel{a_{k}}{\rightarrow} p_{k}
$$

such that $p_{0}, \ldots, p_{k} \in S_{p} \cup\{N I L\}, n_{0}, \ldots, n_{k-1} \in S_{n}, a_{1}, \ldots, a_{k} \in A_{\tau}$. We say that $\sigma$ starts in $p_{0}$ and we write first $(\sigma)=p_{0}$, and also trace $(\sigma)=$ $a_{1} a_{2} \ldots a_{k}$ and last $(\sigma)=p_{k}$. If last $(\sigma)=N I L$, then $\sigma$ is maximal.

- If $k=0$ we define $\mathbf{P}(\sigma)=1$. If $k \geq 1$ we define

$$
\mathbf{P}(\sigma)=\mu\left(p_{0}, n_{0}\right) \cdot \mu\left(p_{1}, n_{2}\right) \cdot \ldots \cdot \mu\left(p_{k-1}, n_{k-1}\right) .
$$


- Let $Q \subseteq S_{p} \cup\{N I L\}$ and $\sigma$ be a finite path in the form written above. If $p_{0}, p_{1}, \ldots, p_{k-1} \in Q$ then we say that $\sigma$ only passes through states in $Q$, and we write $\sigma_{Q}$.

Definition 6. An execution or fullpath is either a maximal execution fragment or an infinite sequence

$$
\pi=p_{0} \leadsto n_{0} \stackrel{a_{1}}{\rightarrow} p_{1} \leadsto n_{1} \stackrel{a_{2}}{\rightarrow} p_{2} \ldots
$$

such that $p_{0}, p_{1}, p_{2}, \ldots \in S_{p}, n_{0}, n_{1}, n_{2} \ldots \in S_{n}, a_{1}, a_{2}, \ldots \in A_{\tau}$. A path is a finite path or a fullpath.

$\operatorname{Path}_{f u l l}(p)$ denotes the set of fullpaths starting in $p$. Similarly, Path fin $(p)$ (Path fin $Q(p)$ for some $Q \subseteq S_{p} \cup\{N I L\}$ ) denotes the set of finite paths starting in $p$ (that only pass through states in $Q$ ). For each process $p, \mathbf{P}$ induces a probability space on $\operatorname{Path}_{f u l l}(p)$ as follows.

Let $\sigma \uparrow$ denote the basic cylinder induced by $\sigma$, that is,

$$
\sigma \uparrow=\left\{\pi \in \operatorname{Path}_{\text {full }}(p): \sigma \leq_{\text {prefix }} \pi\right\},
$$

where $\leq_{\text {prefix }}$ is the usual prefix relation on sequences. We define $\sigma$ Field $(p)$ to be the smallest sigma-field on $\operatorname{Path}_{f u l l}(p)$ which contains all basic cylinders $\sigma \uparrow$ where $\sigma \in \operatorname{Path}_{\text {fin }}(p)$, that is, $\sigma$ ranges over all finite paths starting in $p$. The probability measure Prob on $\sigma$ Field $(p)$ is the unique probability measure with $\operatorname{Prob}(\sigma \uparrow)=\mathbf{P}(\sigma)$.

Lemma 1. (प) Let $p \in S_{p}$ and $\Sigma \subseteq$ Path $_{\text {fin }}(p)$ such that $\sigma, \sigma^{\prime} \in \Sigma, \sigma \neq \sigma^{\prime}$ implies $\sigma \not_{\text {prefix }} \sigma^{\prime}$. Then, $\operatorname{Prob}(\Sigma \uparrow)=\sum_{\sigma \in \Sigma} \mathbf{P}(\sigma)$.

Definition 7. If $p \in S_{p}, L \subseteq A_{\tau}^{*}$ and $C \subseteq S_{p} \cup\{N I L\}$, then $\operatorname{Prob}(p, L, C)=$ $\operatorname{Prob}(\Sigma(p) \uparrow)$, where $\Sigma(p)$ is the set of all finite paths $\sigma$ starting in $p$ with trace in $L$ and with the last process belonging in $C$, that is,

$$
\Sigma(p)=\left\{\sigma \in \operatorname{Path}_{\text {fin }}(p): \operatorname{first}(\sigma)=p, \operatorname{trace}(\sigma) \in L, \text { last }(\sigma) \in C\right\} .
$$

$\operatorname{Path}_{f u l l, Q}(p)$ is defined as the set of fullpaths $\sigma \in \operatorname{Path}_{f u l l}(p)$ such that there is some $k_{\sigma} \geq 0$ and $\sigma^{\prime} \in$ Path $_{\text {fin }, Q}(p)$ such that $\sigma^{\prime}$ is the prefix of $\sigma$ with the length $k_{\sigma}$. Then, in a similar way as above we define a probability space on $\operatorname{Path}_{f u l l, Q}(p)$, the probability measure $\operatorname{Prob}_{Q}\left(\sigma_{Q} \uparrow\right)=\mathbf{P}\left(\sigma_{Q}\right)$ and $\operatorname{Prob}_{Q}(p, L, C)$.

Probabilistic Branching Bisimulation The new result in our approach is a definition of probabilistic branching bisimulation that is weaker than the one in 9 and that can, we think, be extended for probabilistic processes containing nondeterminism. The bisimulation on the set of fully probabilistic graphs we propose is based on the notion of a set of entries (a subset of the set of probabilistic nodes) and a set of exits (a subset of the set of action nodes).

In the following, we introduce the notion of entries and exits for a given graph with equivalence relation $R$ defined on the set of its nodes. An exit of a 
probabilistic node is an action node that is the outgoing node of an external action transition or an internal transition that leads to a new equivalence class. Every probabilistic node has a set of exits. Having the sets of exits determined for each probabilistic node in the graph we can obtain the set of entries. First, the root of the graph is always an entry. Further, an entry in one equivalence class is a node that is first entered from an exit of some other entry by taking either an external or an internal action. In such a way each entry determines the set of its succeeding entries. In other words, a probabilistic node $q$ is not an entry if it is reachable from entries belonging to the equivalence class of $q$ only through internal paths passing through this equivalence class. Finally, for each entry the probabilities for reaching the equivalence classes of its succeeding entries are computed. All entries with the same probability distribution are considered bisimilar. For nodes found not to be entries the probabilities are not computed. Formal definitions follow.

Definition 8 (Entry). If $g$ is a fully probabilistic graph and if $R$ is an equivalence relation on the set of states then:

$\operatorname{Entr} y_{0}(g)=\{\operatorname{root}(g)\}$,

$\operatorname{Entr}_{i+1}(g)=\left\{q: \exists r \in \operatorname{Entr}_{i}: \exists e \in \operatorname{Exit}_{R}(r): e \stackrel{a}{\rightarrow} q, a \in A_{\tau} \& q \notin[r]_{R}\right\}$

$\cup\left\{q: \exists r \in \operatorname{Entr}_{i}: \exists e \in \operatorname{Exit}_{R}(r): e \stackrel{a}{\rightarrow} q, a \in A \& q \in[r]_{R}\right\}$,

where $\operatorname{Exit}_{R}(r)=\left\{s: r \stackrel{\tau^{*}}{\Longrightarrow}[r]_{R} \leadsto s \& \exists C \neq[r]_{R}: s \stackrel{a}{\rightarrow} C, a \in A_{\tau}\right\}$

$\cup\left\{s: r \stackrel{\tau^{*}}{\Longrightarrow}[r]_{R} \sim s \& s \stackrel{a}{\rightarrow}[r]_{R}, a \in A\right\}$.

Finally, Entry $y_{R}(g)=\bigcup_{i>0} \operatorname{Entr} y_{i}(g)$.

$B y \stackrel{\tau^{*}}{\Longrightarrow}$ we denote the transitive and reflexive closure of $\leadsto \cdot \stackrel{\tau}{\rightarrow}$ and $b y \stackrel{\tau^{*}}{\Longrightarrow} Q$ we denote the transitive and reflexive closure of $\left\{p \leadsto \cdot \stackrel{\tau}{\rightarrow} p^{\prime}: p, p^{\prime} \in Q\right\}$ for $Q \subseteq S_{p} \cup\{N I L\}$.

Definition 9. If $g$ is a fully probabilistic graph, $R$ and $\tilde{R}$ are equivalence relations on the set of states such that $\tilde{R} \subseteq R$ and if $r \in S_{p}$, then:

NextEntry $(r)=\left\{q: \exists e \in \operatorname{Exit}_{R}(r): e \stackrel{a}{\rightarrow} q, a \in A_{\tau} \& q \notin[r]_{R}\right\}$

$\cup\left\{q: \exists e \in \operatorname{Exit}_{R}(r): e \stackrel{a}{\rightarrow} q, a \in A \& q \in[r]_{R}\right\}$

and NextEntry $C_{\tilde{R}}(r)=\left\{[q]_{\tilde{R}}: q \in N e x t \operatorname{Entry}(r)\right\}$.

Due to the fact that two entries from the same $R$ equivalence class may have different sets of exits and sets of next entries, these sets have to be parametrized by the entry they are associated to (see Example 2 .

Definition 10 (Probabilistic Branching Bisimulation). Let $g$ and $h$ be fully probabilistic graphs. If $R$ is an equivalence relation on $S_{g} \cup S_{h} \cup\left\{N I L_{g}, N I L_{h}\right\}$ such that:

0. $(\operatorname{root}(g), \operatorname{root}(h)) \in R$;

1. if $(p, q) \in R$ and $p \sim s$ then either

$1.0(s, q) \in R$ or 
1.1 there are $v, t$ such that $(p, v),(s, t) \in R$ and

$q \stackrel{\tau^{*}}{\Longrightarrow} v \leadsto t$ or $q \stackrel{\tau}{\rightarrow} \cdot \stackrel{\tau^{*}}{=} v \leadsto t$

2. if $(p, q) \in R$ and $p \stackrel{a}{\rightarrow} s$ then either

$2.0 a=\tau$ and $(s, q) \in R$ or

2.1 there are $v, t$ such that $(q, v),(s, t) \in R$ and

$q \stackrel{\tau^{*}}{\longrightarrow} \cdot \sim v \stackrel{a}{\rightarrow}$ tor $q(\stackrel{\tau}{\rightarrow} \cdot \sim)^{*} v \stackrel{a}{\rightarrow} t ;$

3. there is an equivalence relation $\tilde{R}$ on $\operatorname{Entr} y_{R}(g) \cup \operatorname{Entr} y_{R}(h)$ such that $\tilde{R} \subseteq R$ and

3.0. $(\operatorname{root}(g), \operatorname{root}(h)) \in \tilde{R}$;

3.1. if $(p, q) \in \tilde{R}$ then for any $C \in N$ extEntry $C_{\tilde{R}}(p) \cup N e x t E n t r y C_{\tilde{R}}(q)$ and for any $a \in A$,

$\operatorname{Prob}_{[p]_{R}}\left(p, \tau^{*}, C\right)=\operatorname{Prob}_{[q]_{R}}\left(q, \tau^{*}, C\right)$ and

$\operatorname{Prob}_{[p]_{R}}\left(p, \tau^{*} a, C\right)=\operatorname{Prob}_{[q]_{R}}\left(q, \tau^{*} a, C\right)$;

then $(R, \tilde{R})$ is a probabilistic branching bisimulation relation between $g$ and $h$.

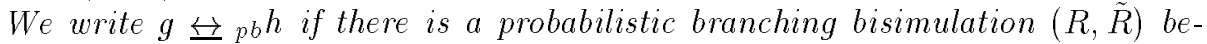
tween $g$ and $h$.

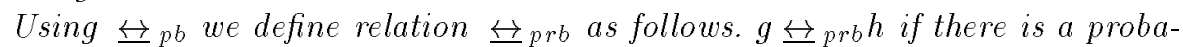
bilistic branching bisimulation $(R, \tilde{\tilde{R}})$ between $g$ and $h$ such that $\{\operatorname{root}(g)$, root $(h)\}$ is an $R$ equivalence class and if root $(g) \sim s$ then there is $t$ such that root $(h) \sim t$ and $(s, t) \in R$, and vice versa. We say that $g$ and $h$ are probabilistically rooted branching bisimilar. The condition above is called probabilistic rooted branching condition.

From now on, instead of $\operatorname{Prob}_{[p]_{R}}\left(p, \tau^{*}, C\right)$ and $\operatorname{Prob}_{[p]_{R}}\left(p, \tau^{*} a, C\right)$ we will write $\operatorname{Prob}_{R}\left(p, \tau^{*}, C\right)$ and $\operatorname{Prob}_{R}\left(p, \tau^{*} a, C\right)$, respectively. (From $\operatorname{Prob}_{R}\left(p, \tau^{*}, C\right.$ ) it is clear that $[p]_{R}$ is the subscript set in the original notation.) Even if $[p]_{\tilde{R}}$ is not a NextEntry class for $p$, we still take $\operatorname{Prob}_{[p]_{R}}\left(p, \tau^{*},[p]_{\tilde{R}}\right)=1$.

Example 1. Let $g$ and $h$ be fully probabilistic graphs given in Figure 3 We define the following equivalence relation: $R=\{\{1,2,3,4,5\},\{6,8\},\{7,9\},\{N I L\}\}$ Then Entry $y_{R}(g \cup h)=\{1,3, N I L\}$ and we define $\tilde{R}=\{\{1,3\},\{N I L\}\}$.
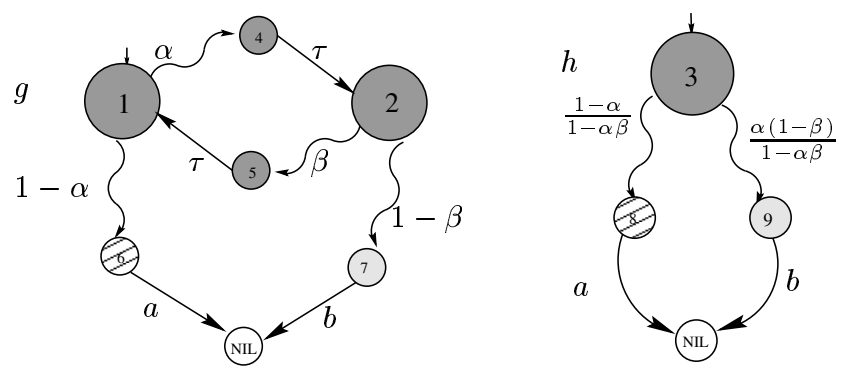

Fig. 3. Bisimilar graphs from example 1. 
Probabilities of these entries to the $\tilde{R}$ equivalence classes are given in the following table. In the table we put - in the $(r, C)$ field if $C \notin N e x t E n t r y C_{\tilde{R}}(r)$. We omit the row of the NIL entry.

\begin{tabular}{||c||c|c||c||c|c||c||c|c|}
\hline$\tau^{*}$ & $\{1,3\}$ & $\{N I L\}$ & $\tau^{*} a$ & $\{1,3\}$ & $\{N I L\}$ & $\tau^{*} b$ & $\{1,3\}$ & $\{N I L\}$ \\
\hline 1 & 1 & 0 & 1 & - & $\frac{1-\alpha}{1-\alpha \beta}$ & 1 & - & $\frac{\alpha(1-\beta)}{1-\alpha \beta}$ \\
\hline 3 & 1 & 0 & 3 & - & $\frac{1-\alpha}{1-\alpha \beta}$ & 3 & - & $\frac{\alpha(1-\beta)}{1-\alpha \beta}$ \\
\hline
\end{tabular}

Thus, $(R, \tilde{R})$ is a probabilistic branching bisimulation between $g$ and $h$.

Example 2. The following example shows that the root condition as it is given is sufficient.

Let $g$ and $h$ be graphs given in Figure $4 \mathrm{R}=\{\{1,3\},\{2,4,5,6\},\{\mathrm{NIL}\}\}$ is a rooted branching bisimulation between $g$ and $h$ (it is the only one). But $N e x t E n t r y y_{R}(1)=\{2\}$ and $N e x t E n t r y_{R}(3)=\{N I L\}$ from which we conclude that $\tilde{R}$ cannot be defined, that is, a probabilistic branching bisimulation between $g$ and $h$ does not exist.

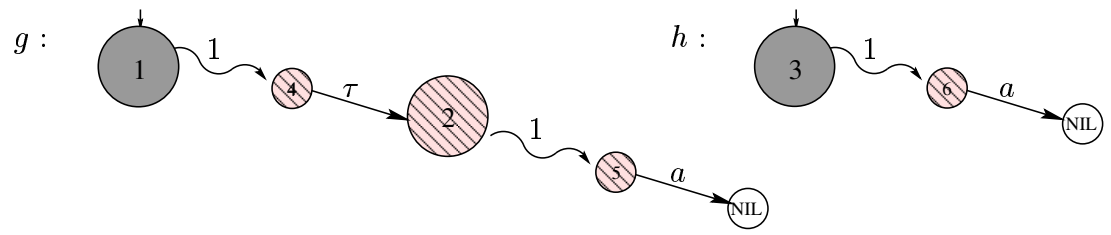

Fig. 4. Bisimilar graphs from example 2.

Example 3. Let $g$ and $h$ be graphs given in Figure 5 We define the following relation: $R=\{\{1,2,4,6,8,9\},\{3,5,7,12,15,18\},\{10,13,16\},\{11,14,17\}\}$. Then

\begin{tabular}{|c|c|c|c|c|c|c|c|}
\hline$r$ & 1 & 3 & 2 & 4 & 5 & 6 & 7 \\
\hline $\operatorname{Exit}_{R}(r)$ & 10,11 & 12 & 10,11 & 13,14 & 15 & 16,17 & 18 \\
\hline NextEntry $y_{R}(r)$ & 3 & 2 & 3 & 5 & 6 & 7 & 6 \\
\hline
\end{tabular}

$\operatorname{Entr}_{R}(g \cup h)=\{1,2,3,4,5,6,7\}$. If we take $\tilde{R}_{0}=\{\{1,2,4,6\},\{3,5,7\}\}$, then, for instance, 1 and 2 do not have same probabilities, which means $\left(R, \tilde{R}_{0}\right)$ is not probabilistic branching bisimulation between $g$ and $h$. But if we refine classes of $\tilde{R}_{0}$ into $\tilde{R}_{1}$ in the following way: $\tilde{R}_{1}=\{\{1,4\},\{2,6\},\{3,5,7\}\}$, then it is easy to check that $\tilde{R}_{1}$ satisfies the third requirement in Definition III We conclude that $\left(R, \tilde{R}_{1}\right)$ is a probabilistic branching bisimulation between $g$ and $h$.

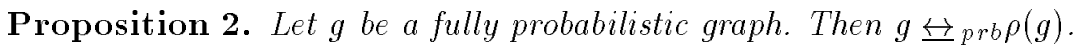



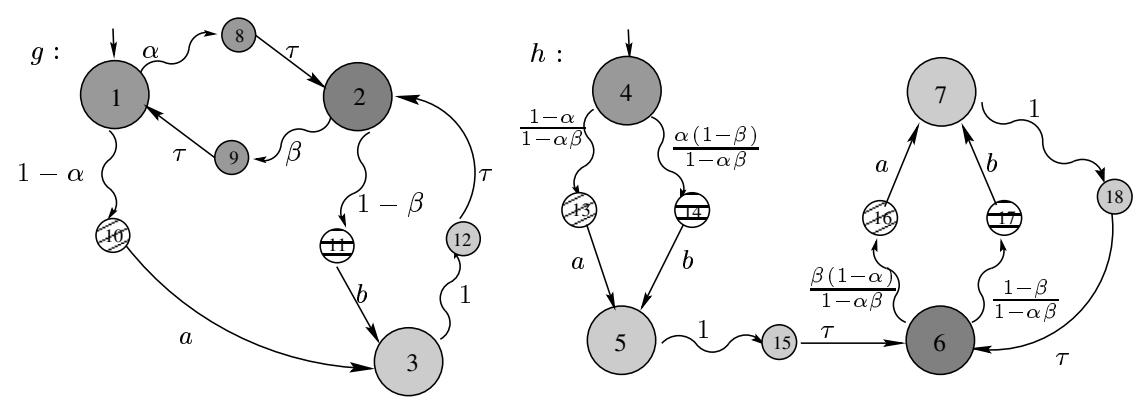

Fig. 5. Bisimilar graphs from example 3 .

Proposition 3. Let $(R, \tilde{R})$ be a probabilistic branching bisimulation between $g$ and $h$. If $p, q \in S_{g} \cup S_{h}$ and $(p, q) \in \tilde{R}, N e x t E n t r y C_{\tilde{R}}(p)=N e x t E n t r y C_{\tilde{R}}(q)$.

Corollary 1. Let $(R, \tilde{R})$ be a probabilistic branching bisimulation between $g$ and $h$. If $p \in \operatorname{Entr}_{R}(g)$ then there is a node $q$ in $h$ such that $(p, q) \in \tilde{R}$.

Proposition 4. If $R$ is an equivalence relation on the fully probabilistic graph $g$ and if $C$ is an $R$ equivalence class containing a probabilistic node then there is an entry node in $C$.

Using the results from the previous propositions, the Congruence theorem can be proved.

Theorem 1. $\leftrightarrow$ prb is a congruence relation on $\mathbf{G}$ with respect to the probabilistic choice operator, the sequential composition and the abstraction operator.

Proof. The proof about the abstraction operator is based on the relation between the set of entries in the original graph and the one obtained with abstraction. Namely, the second set is a subset of the first set. Having this in mind, a relation between probabilities of the entries in the two graphs can be established. (For more details see 4.) For the two other operators it is not difficult to construct a probabilistic bisimulation relation on composed graph from already existing relations on the components (in that composition). Namely, for the sequential composition the only interesting detail is the merging the NIL equivalence class from the first component with the equivalence class of the root of the second component. Two cases occur depending on NIL being an entry or not in the first graph. The part concerning the probabilistic choice operator can easily be proved.

Theorem 2 (Soundness theorem). G/ $\leftrightarrow$ prb is a model of the presented fully probabilistic process algebra with the verification rules $P V R 1, P V R 2, \ldots$. 


\section{Deciding the Branching Bisimulation Equivalence}

In this section we present an algorithm that computes a probabilistic branching bisimulation equivalence relation for given fully probabilistic graphs. Namely, the algorithm decides if the root nodes of the graphs have the same branching structure and, further, if they have the same probability measures. At the end it returns a pair of relations that relates these graphs if such relations exist. The basic idea of the algorithm is to start with the coarsest branching bisimulation relation that relates two nodes if and only if they have the same branching structure, regardless of their probability measures. In Definition 10 one can notice that probabilistic transitions in the part which concerns the branching structure (items 0, 1 and 2) can be viewed as internal transitions. This gives us liberty to employ any algorithm that decides branching bisimulation on non-probabilistic systems. In particular, here we use the algorithm for deciding branching bisimulation equivalence in 10 . The original algorithm is defined on one graph in which case the output is the coarsest branching bisimulation on that graph, since it always exists. The algorithm can slightly be modified into an algorithm that works on a union of two graphs (which is what we need). In this case (Step1) the output is either the branching bisimulation equivalence relation $R$ between the two graphs with roots root 1 and root ${ }_{2}$, and it is the input of the second part of the algorithm; or it has found that the two graphs are not branching bisimilar (the root nodes are not $R$-related) and it returns the empty relation meaning that two graphs are not branching bisimilar. In the latter case the given graphs are not probabilistically branching bisimilar as well (Step2). Before the second part is run, the set of entries w.r.t. $R$ is calculated (Step3). The second part of the algorithm concerns probabilities. Starting from the $R$ equivalence classes restricted on the entries as the initial value for $\tilde{R}$ (Step4, where $B B$ is the partition induced by $R$ ), the algorithm refines the $\tilde{R}$ equivalence classes by comparing the probability measures for the nodes belonging to the same class (Step5). If it has been found that two or more nodes from the same equivalence class have different probabilities, then it is split into separate subclasses. Finally, if it has been detected that the roots have been split apart then the algorithm terminates (Step6) with the conclusion that the two graphs are not probabilistically bisimilar (returning the pair $(\emptyset, \emptyset)$ ). Otherwise, the algorithm returns the pair of relations that makes graphs $g$ and $h$ probabilistically branching bisimilar $(S t e p 7)$. The crucial point here is the definition of a splitter. (Note: many algorithms concerning bisimulation are based on a notion of a splitter defined in an appropriate way for that particular relation.)

Definition 11. Let $g$ be a fully probabilistic graph and $R$ an equivalence relation on $g$. Let $\tilde{R}$ be an equivalence relation that is a subset of $R$. And let $\Pi$ be the partition induced by $\tilde{R}$. A pair $(a, C)$ for $a \in A_{\tau}$ and $C \in \Pi$ is a splitter of $\Pi$ if for some $E \in \Pi$ and $p, p^{\prime} \in E$, if $C \in N$ extEntry $C_{\Pi}(p)$ or $C \in N$ NextEntryC $C_{\Pi}\left(p^{\prime}\right)$ then

$$
\operatorname{Prob}_{R}\left(p, \tau^{*} a, C\right) \neq \operatorname{Prob}_{R}\left(p^{\prime}, \tau^{*} a, C\right) .
$$


Thus a splitter $(a, C)$ of a partition $\Pi$ indicates a class in $\Pi$ such that contains states which prevent $(R, \Pi)$ from being a probabilistic branching bisimulation. Moreover, it indicates that partition $\Pi$ has to be refined to $\Pi^{\prime}$ in such a way that $(a, C)$ is not a splitter of $\Pi^{\prime}$. And thus, we split the set of entries in finer classes, subsets of corresponding $R$ classes, until we obtain a partition (the $\tilde{R}$ relation) that meets the third requirement in Definition 10 Formally,

Definition 12. Let $g, R$ and $\Pi$ be defined like in the previous definition and let $(a, C)$ be a splitter of $\Pi$. If $E \in \Pi$ we define a refinement of $E$ w.r.t. $(a, C)$, Re fine $(E, a, C)$, in the following way:

for some set of indices $N$ such that

$$
\operatorname{Refine}(E, a, C)=\left\{E_{n}: n \in N\right\},
$$

1. $\left\{E_{n}: n \in N\right\}$ is a partition of $E$ and

2. $\forall n \in N: \forall s, t \in E_{n}: \operatorname{Prob}_{R}\left(s, \tau^{*} a, C\right)=\operatorname{Prob}_{R}\left(t, \tau^{*} a, C\right)$.

The refinement of $\Pi$ w.r.t. splitter $(a, C)$ is:

$$
\operatorname{Refine}(\Pi, a, C)=\bigcup_{E \in \Pi} \operatorname{Refine}(E, a, C) \text {. }
$$

The probabilities $\operatorname{Prob}_{R}\left(p, \tau^{*} a, C\right)$ can be computed by solving the linear equation system (see e.g. $\times 9$ )

$$
\begin{array}{ll}
x_{p}=1 & \text { if } a=\tau \text { and } p \in C \\
x_{p}=0 & \text { if } \text { Path }_{f u l l,[p]_{R}}\left(p, \tau^{*} a, C\right)=\emptyset \\
x_{p}=\sum_{t \in[p]_{R}} \mathbf{P}(p, \tau, t) \cdot x_{t}+\mathbf{P}(p, a, C) & \text { otherwise }
\end{array}
$$

The algorithm is given step-by-step in Figure 6 The input is given as a union

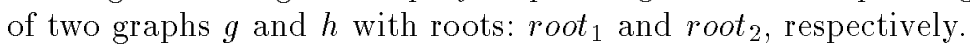

Input: finite fully probabilistic graphs $g$ and $h$ with $\left(S \sim, \rightarrow, \mu\right.$, root $_{1}$, root $\left._{2}\right)$

Output : $(R, \Pi)$ probabilistic branching bisimulation between $g$ and $h$ if it exists $(\emptyset, \emptyset)$ if $g$ and $h$ are not probabilistically branching bisimilar

Method:

Step1: Call the coarsest branching bisimulation relation algorithm for the graphs $g$ and $h$, and receive $R$;

Step2: If $R=\emptyset$ then Return $(\emptyset, \emptyset)$;

Step3: Compute the sets: Entry, NextEntry $(r)$;

Step $4: \quad \Pi:=\left\{E \cap E_{\text {Entry }}: E \in B B\right\} \backslash\{\emptyset\}$

Step5: While $\Pi$ contains a splitter $(a, C)$ do $\Pi:=\operatorname{Refine}(\Pi, a, C)$;

Step6: If root $_{1}$ and root $_{2}$ are not $\Pi$-related then Return $(\emptyset, \emptyset)$;

Step7 : Return $(R, \Pi)$.

Fig. 6. Algorithm for computing probabilistic branching bisimulation.

Lemma 2. The algorithm can be implemented in polynomial time in the number of states $n$. 
Proof. Let $g$ and $h$ be finite fully probabilistic graphs with $n$ states and $m$ transitions (total number of states and transitions for both graphs).

For the first part of the algorithm, finding the coarsest bisimulation relation we use the algorithm in 10 which has time complexity $\mathcal{O}(n \cdot m)$. In this step the probabilistic transitions are treated as internal transitions. The set of entries with respect to $R$ can be found with a depth first search with the algorithm in I (with time complexity $\mathcal{O}(m)$ ).

The second part of the algorithm consists of solving the system of linear equations and refining the current partition with respect to a found splitter. The test whether $\operatorname{Path}_{f u l,[p]_{R}}\left(p, \tau^{*} a, C\right)=\emptyset$ can be done by a reachability analysis of the underlying directed graph. In the worst case we have to repeat the refinement step $n$ times. And in each of them we have to solve a system of linear equations with $n$ variables and $n$ equations which takes $\mathcal{O}\left(n^{2.8}\right)$ time with the method in 1. Thus we obtain the time complexity of the second part of the algorithm to be in the worst case $\mathcal{O}\left(n^{3.8}\right)$.

In total since $m \leq n^{2} \cdot\left|A_{\tau}\right|$ we obtain $\mathcal{O}\left(n^{3.8}\right)$ time complexity of the algorithm.

\section{Conclusion}

In this paper we presented a version of fully probabilistic process algebra with abstraction which contains, in addition to the axioms for the basic operators, a set of verification rules. These rules tie in successfully the idea of abstraction in process algebra with the results from Markov chain analysis. Furthermore, we proposed a probabilistic branching bisimulation relation which corresponds to this process algebra in the sense that it gives a model for it. In such a way we obtain a model for the verification rules. One of the advantages of having such rules is that they give the probability distribution after abstraction from the internal actions, which in the model (i.e. using the bisimulation relation) should be calculated separately.

Due to the absence of non-determinism in the algebra we did not incorporate parallel composition since the model that we had used for parallel composition in the previous work requires non-determinism. Nevertheless the process algebra proposed in this paper can be widely applied. Namely, in $>3$ one can find examples of protocols for which the protocol specification does not contain non-determinism. Thus, the techniques from this paper can be applied to these specifications for the verification part, that is, for proving that these protocols behave with probability 1 as a one place buffer. Moreover, the way we presented the definitions in the paper left room for an extension with non-determinism. For example, the definition of the bisimulation relation is given for the alternating model which essentially includes non-determinism. Thus, we think that this work is good start for obtaining a probabilistic branching bisimulation relation for probabilistic processes that contain non-determinism. 


\section{References}

1. A. Aho, J. Hopcroft, J. Ullman, The design and analysis of computer algorithms, Addison-Wesley Publishing Company, 1974.

2. S. Andova, Process algebra with probabilistic choice (extended abstract), Proc. ARTS'99, Bamberg, Germany, J.-P. Katoen, ed., LNCS 1601, Springer-Verlag, pp. 111-129, 1999. Full version report CSR 99-12, Eindhoven University of Technology, 1999.

3. S. Andova, Process algebra with interleaving probabilistic parallel composition, Eindhoven University of Technology, CSR 99-04, 1999.

4. S. Andova, J.C.M. Baeten, Abstraction in probabilistic process algebra (extended abstract), http://www.win.tue.nl/ ^ suzana.

5. J.C.M. Baeten, J.A. Bergstra, J.W. Klop, On the consistency of Koomen's fair abstraction rule, Theor. Comp. Sci. 51, pp.129-176, 1987.

6. J.C.M. Baeten, J.A. Bergstra, S.A. Smolka, Axiomatizing probabilistic processes: ACP with generative probabilities, Information and Computation 121(2), pp. 234255, Sep. 1995.

7. J.C.M. Baeten, W. P. Weijland, Process algebra, Cambridge University Press, 1990.

8. C. Baier, On algorithmic verification methods for probabilistic systems, Habilitation thesis, Univ. Mannheim, 1998.

9. C. Baier, H. Hermanns, Weak bisimulation for fully probabilistic processes, Proc. CAV'97, LNCS 1254, pp. 119-130, 1997.

10. J.F. Groote, F. Vaandrager, An efficient algorithm for branching bisimulation and stuttering equivalence, Proc. ICALP'90, LNCS 443, pp. 626-638, 1990.

11. C.-C. Jou, A spects of probabilistic process algebra, Ph.D.Thesis, State University of New York at Stony Brook, 1990. 